\title{
1 A draft genome of Alliaria petiolata (garlic 2 mustard) as a model system for invasion 3 genetics \\ 4
}

$5 \quad$ Nikolay Alabi ${ }^{1}$, Yihan Wu ${ }^{1}$, Oliver Bossdorf ${ }^{2}$, Loren H. Rieseberg ${ }^{3}$ 6 and Robert I. Colautti ${ }^{1 *}$

7

$8 \quad{ }^{1}$ Biology Department, QueEn's University, Kingston, OntARIO, CANAdA

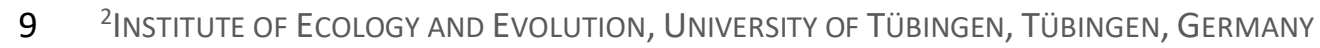

10 32Department of Botany and Biodiversity Research Centre, University of British Columbia, Vancouver, BC, 11 CANADA

12

13

CORRESPONDING AUTHOR: ROBERT.COLAUTTI@QUEENSU.CA

14

15

16

17 Keywords: Alliaria petiolata, EFCC3, invasion genetics, garlic mustard, Illumina, mate pairs 


\section{Abstract}

The emerging field of invasion genetics examines the genetic causes and consequences of biological invasions, but few study systems are available that integrate deep ecological knowledge with genomic tools. Here we report on the de novo assembly and annotation of a genome for the biennial herb Alliaria petiolata (M. Bieb.) Cavara \& Grande (Brassicaceae), which is widespread in Eurasia and invasive across much of temperate North America. Our goal was to sequence and annotate a genome to complement resources available from hundreds of published ecological studies, a global field survey, and hundreds of genetic lines maintained in Germany and Canada. We sequenced a genotype (EFCC-3-20) collected from the native range near Venice, Italy and sequenced paired-end and mate pair libraries at $~ 70 \times$ coverage. A de novo assembly resulted in a highly continuous draft genome $\left(N_{50}=121 \mathrm{Mb} ; L_{50}=2\right)$ with $99.7 \%$ of the $1.1 \mathrm{~Gb}$ genome mapping to contigs of at least $50 \mathrm{~Kb}$ in length. $A$ total of 64,770 predicted genes in the annotated genome include $99 \%$ of plant BUSCO genes and $98 \%$ of transcriptome reads. Consistent with previous reports of (auto)hexaploidy in western Europe Almost, we found that almost one third of BUSCO genes (390/1440) mapped to two or more scaffolds despite a genome-wide average of $<2 \%$ heterozygosity. The continuity and gene space quality of our draft genome assembly will enable genomic studies of $A$. petiolata to address questions relevant to invasion genetics and conservation efforts.

\section{Introduction}

Biological invasions are a threat to global biodiversity with significant impacts to human health and welfare (Mack et al., 2000). They also present opportunities for large-scale 'natural' experiments to study ecological and evolutionary processes in the wild (Mooney and Cleland, 2001; Sax et al., 2005). Despite a large body of ecological research and a growing number of evolutionary studies of invasive species, functional genetic and 'omics approaches have been rare among studies of invasive species until recently (reviewed in (Barrett, 2015; Bock et al., 2015); but see e.g. (Barrett et al., 2016; Boheemen et al., 2017; Bourne et al., 2020)). Apart from studies using neutral markers to assess population structure (reviewed in (Dlugosch and Parker, 2008)), relatively little is known about the genetic causes and consequences of biotic invasions at the molecular level. A lack of genomic resources has hindered high-resolution genetic studies of most invasive species. Here, we report on a draft genome for the herbaceous biennial plant Alliaria petiolata, a plant invader in North America with potential to become a model system for the emerging field of invasion genetics.

Several factors favour A. petiolata as an emerging model system for invasion genetics (Colautti et al., 2014). First, it is widely distributed with variable ecological impacts throughout its range (Lankau et al., 2009; USDA, 2020). Second, it has a relatively simple two-year life cycle with well-defined life stages (seed, rosette, bolting, senescent). This simple life history facilitates measurements of lifetime fitness, natural selection, and their impacts on population dynamics in natural populations. Third, A. petiolata is a member of the Brassicaceae and therefore benefits from genetic resources available for well-studied species like Brassica rapa (canola) and the model plant Arabidopsis thaliana, providing opportunities for functional and comparative genomics. Fourth, high selfing rates produce naturally inbred seed families that can be maintained through single-seed descent. Fifth, A. petiolata has been the focal species in hundreds of field surveys and experimental studies, including influential studies testing the role of natural enemies (Lewis et al., 2006), competition (Prati and Bossdorf, 2004), the 'novel weapons' hypothesis (Callaway et al., 2008), competitive ability (Bossdorf et al., 2004), glucosinolate metabolism 
(Haribal et al., 2001), and eco-evolutionary dynamics (Lankau et al., 2009). One mechanism that is especially well-studied is the production of secondary metabolites and their effects on soil microbiota, particularly the suppression of mycorrhizal fungi that form beneficial symbiotic networks among native plant roots (Anthony et al., 2017; Duchesneau et al., 2020). Understanding the genomic basis of such interactions with soil microbes will not only advance basic science but it also has potential applications in plant restoration and agriculture.

Recent efforts to develop A. petiolata as a model system include the Global Garlic Mustard Field Survey (GGMFS), which mobilized 164 participants from 16 countries across North America and Europe to collect field data and seed samples across Europe and North America, resulting in thousands of seed families from 383 distinct populations (Colautti et al., 2014). A subset of inbred lines collected across North America have been maintained through single-line descent in labs in Germany (Bossdorf Lab, University of Tübingen) and Canada (Colautti Lab, Queen's University). Adding to these resources, we here report on a draft genome of a single $A$. petiolata genotype from Europe, annotated with RNA sequencing of leaf and root tissue.

\section{Methods \& Materials}

\section{Study Organism and Line Derivation}

Alliaria petiolata (M. Bieb.) Cavara \& Grande is a biennial herbaceous plant in the Thlaspideae tribe of the Brassicaceae family. It was introduced to North America prior to 1868, when it was discovered in Long Island, New York (Nuzzo, 1993). By 1948 it was reported on the West Coast of North America and has established in at least 37 U.S. States and five Canadian provinces from the Atlantic coast to the Pacific (Cavers et al., 1979; USDA, 2020). As the only species of Alliaria with a broad distribution, $A$. petiolata is relatively easy to identify in natural habitats owing to its white flowers and dentated peltate leaves with long petioles. It is considered a noxious weed across most of its introduced range, due in part to impacts on native plants and tree regeneration in deciduous forest ecosystems (Cipollini and Cipollini, 2016; Stinson et al., 2007). Two genome types have been identified, including diploids ( $2 n=$ 14) in Eastern Europe and Western Asia and hexaploids ( $2 n=42$ and $2 n=36$ ) in Central Europe and North America (Esmailbegi et al., 2018; Weiss-Schneeweiss and Schneeweiss, 2003).

\section{So generation}

All source material for genome and transcriptome sequencing originated from a single individual grown from seed collected as part of the GGMFS (Colautti et al., 2014). The inbred line used in this study is from population EFCC3, collected in 2011 from a small forest fragment $\left(\sim 2,000 \mathrm{~m}^{2}\right)$ surrounded by agricultural land, about $75 \mathrm{~km}$ northwest of Venice, Italy (UTM $45.71^{\circ} \mathrm{N} \times 11.72^{\circ} \mathrm{E}$ ). The specific seed line was sampled at $3.2 \mathrm{~m}$ along a $10 \mathrm{~m}$ sampling transect originating at the edge of population EFCC3. This inbred line (code EFCC3-3-20) is currently maintained along with other GGMFS seed collectionsby two of the coauthors in replicate collections in Tübingen, Germany (Bossdorf) and Kingston, Ontario, Canada

95 (Colautti). In July 2012, ten seeds of the EFCC3-3-20 genotype from the original $\mathrm{S}_{0}$ field collection were removed from cold storage $\left(4^{\circ} \mathrm{C}\right)$, surface washed with a mild detergent and rinsed with distilled $\mathrm{H}_{2} \mathrm{O}$ before surface sterilizing in $10 \%$ bleach for 10 minutes. Sterilized seeds were again rinsed with distilled $\mathrm{H}_{2} \mathrm{O}$ 
We stratified seeds in the dark at $10{ }^{\circ} \mathrm{C}$ for $\sim 90$ days and thereafter inspected weekly until emerging radicles were observed. Germinating seeds were transplanted into 4" plastic pots containing a peat soil mixed with vermiculite that was watered to saturation and placed under shade cloth in the Horticulture Greenhouses at the University of British Columbia. We let seedlings establish in soil, watered as needed, for four weeks before a small amount ( $5 \mathrm{~mm} \times 2 \mathrm{~mm}$ ) of young leaf meristem tissue was harvested from a single individual and immediately preserved in liquid nitrogen. Roughly 25 to $50 \mathrm{~mm}^{3}$ of this tissue was divided into two separate $2 \mathrm{~mL}$ screw-cap tubes, each containing two stainless steel ball bearings of $2 \mathrm{~mm}$ diameter. Tubes were flash-frozen in liquid nitrogen and used for genomic DNA purification and genome sequencing.

A second individual from the same inbred family was transplanted to an 8" plastic pot and fertilized with 20/20/20 N/P/K fertilizer to encourage rosette growth before being moved outside from October 2012 to April 2013 for cold vernalization at the University of British Columbia Horticulture Greenhouses. In April 2013, we moved the plant back into the greenhouse and sprayed with $2 \%$ insecticidal soap to remove pests. Once inside the greenhouse, the plant was left to mature and set seed autonomously via self-pollination. Mature siliques were harvested in July 2013 and seeds were stored in paper envelopes at $4{ }^{\circ} \mathrm{C}$.

\section{$\mathrm{S}_{2}$ generation}

In May 2016, we removed a subset of 10 seeds of the $S_{1}$ generation from cold storage and germinated in a $60 \mathrm{~mm} \times 15 \mathrm{~mm}$ petri dish containing filter paper covered with a mixture of autoclaved ProMix soil and silica sand (1:9 ratio). We added distilled water until saturation and thereafter petri dishes were sealed with paraffin wax before storing in the dark at $4^{\circ} \mathrm{C}$. Of these, six seedlings germinated and were retained for transcriptome sequencing, divided into one of two treatments. The first true leaf from each of the three plants in the experimental treatment were cut with scissors. We used a Kimwipe tissue saturated with either $0.4 \mathrm{mM}$ jasmonic acid $(\mathrm{JA})$ dissolved in $10 \%$ ethanol (treatment) or $10 \%$ ethanol alone (control), adhered directly to maintain contact the cut site (treatment) or uncut leaf (control). We replaced the saturated Kimwipe every $8 \mathrm{~h}$ to maintain the signal. After $48 \mathrm{~h}$ of treatment, we harvested seedlings and preserved treated leaves and root tissue in liquid nitrogen, to be used for RNA purification and transcriptome sequencing.

\section{DNA Isolation \& Library Construction}

In September 2013, frozen tissue from the $S_{1}$ genotype was pulverized and extracted using a Cetyltrimethyl Ammonium Bromide (CTAB) protocol (Clarke, 2009) with the following modifications. After pulverising tissue in a bead mill homogenizer at $60 \mathrm{~Hz}$ for $60 \mathrm{~s}$, we added $1 \mathrm{~mL}$ chilled wash buffer (200mM Tris- $\mathrm{HCl}$ pH 8.0, 50mM EDTA, $250 \mathrm{mM} \mathrm{NaCl}$ ) and incubated on ice for $10 \mathrm{~min}$. The purpose of this wash step is to remove secondary metabolites after disruption of cell walls but prior to cell lysis with $\mathrm{CTAB}$. Following this initial wash step, we spun tubes in a microcentrifuge at $4000 \mathrm{~g}$ and $4^{\circ} \mathrm{C}$ for 10 minutes, then discarded the supernatant and added another $1 \mathrm{~mL}$ of wash buffer. This was repeated once more for a total of three wash cycles until no coloration was visible in the supernatant. After final discard of the supernatant and addition of warm lysis buffer as per the CTAB protocol, we vortexed tubes briefly to resuspend plant cells. After completion of the CTAB protocol, pellets were dissolved in $50 \mu \mathrm{L}$ of reverse osmosis (RO) $\mathrm{H}_{2} \mathrm{O}$ and sent to Centre d'expertise et de services Génome Québec (Génome Québec) for library preparation and sequencing. 
We used four separate sequencing libraries for genome assembly: (i) One whole-genome shotgun sequencing library using the Illumina TruSeq DNA v1 preparation kit with a target fragment length of 150b. (ii \& iii) Two Illumina Nextera MatePair libraries with target lengths of $5 \mathrm{~Kb}$ and $10 \mathrm{~Kb}$. These three libraries were multiplexed and sequenced on a single flowcell of Illumina HiSeq 2000 using $2 \times 100 b$ paired end (PE) sequencing chemistry. (iv) Target fragment lengths of $450 \mathrm{~b}$ using the Illumina TruSeq DNA v1 and sequenced on Illumina MiSeq with $2 \times 250 \mathrm{~b}$ paired-end reads.

\section{RNA Isolation \& Library Construction}

For RNA purification, we pulverized frozen leaf and root tissue In March 2017, in the same manner as the DNA extraction protocol outlined in the previous section. After pulverizing the tissue, we extracted whole RNA from each plant separately using Invitrogen's TRIzol reagent, following the manufacturer's protocol (Pub No. MAN0001271 Rev. A.0). We sequenced four of the six extractions with the highest RNA yields at Génome Québec using the Illumina TruSeq LT kit and multiplex kit for sequencing on a single lane of Illumina MiSeq with $2 \times 125 \mathrm{~b}$ paired-end reads. Four separate libraries were sequenced based on tissue and treatment: Control Leaf $(\mathrm{CL})$, Control Root (CR), Treated Leaf (TL), and Treated Root (TR).

\section{Data Processing Methods}

Raw sequencing data was processed and demultiplexed by Génome Québec, and copied to the Frontenac cluster hosted by the Centre for Advanced Computing (CAC) at Queen's University and the Cedar cluster maintained by Simon Fraser University on behalf of Compute Canada. The CAC maintains the Rosalind Franklin Cluster for Analysis of Complex Genomes, which is a 256-core computing cluster with 2 TB of RAM. We used this hardware for the memory-intensive steps de novo genome assembly, with the remaining analyses completed using shared Frontenac and Cedar clusters, and on personal computers. All FASTQ files from both experiments passed quality controls using fastqc (version 0.11.5) (Andrews, 2010). We used the raw, demultiplexed FASTQ files for de novo assembly, but the transcriptome data were pre-processing using cutadapt (Martin, 2011, p. 201) to trim adapters and removing quality reads shorter than $25 \mathrm{~b}$ prior to assembly.

Our genome assembly pipeline involved two main steps. First, we used ALLPATHS-LG (Gnerre et al., 2011) version R52488 to assemble contigs from both the HiSeq and MiSeq paired-end libraries and then to link contigs into scaffolds using the $5 \mathrm{~Kb}$ and $10 \mathrm{~Kb}$ MatePair libraries. The analysis parameters included PLOIDY = 2 and HAPLOIDIFY = TRUE to perform a diploid genome assembly. Although our genome is likely hexaploidy, polyploid models are not supported by ALLPATHS-LG and low heterozygosity is expected given the high selfing rates in natural populations. Second, we joined scaffolds from ALLPATHS-LG into larger mega-scaffolds using redundans (Pryszcz and Gabaldón, 2016) version $0.13 \mathrm{c}$, with the following parameters: identity $=0.9$, iters $=5$, joins $=5$, limit $=1$, linkratio $=0.7$, mapq $=10$, minlength $=1000$ and overlap $=0.75$. We repeated this script four times with output scaffolds of the prior run acting as input scaffolds given the long run-time required ( $28 \mathrm{~d})$. This second combines scaffolds with overlapping similarity, resulting in mega-scaffolds that can span across multiple chromosomes.

Sequencing data from the transcriptome experiment were cleaned and assembled with trinity (Grabherr et al., 2011) following protocols outlined on the software documentation and in Haas et al (Haas et al., 2013). We used default parameters and the quality of the assembly was analyzed using the custom perl scripts included in the trinity package to examine full length transcripts and Contig Nx lengths (i.e. 
analyze_blastPlus_topHit_coverage.pl, TrinityStats.pl). Additionally, we mapped read pairs to the transcriptome assembly to assess read content using bowtie2 (version 2.3.3.1) with default parameters (Langmead and Salzberg, 2012). We used Transdecoder to predict open reading frames in transcripts before using Trinotate to annotate and analyze assembled transcripts (Haas et al., 2013).

As a first step in annotation, we established a detailed repeat library. Miniature Inverted Transposable Elements (MITES) represent the most abundant transposable elements in plants and were identified using MITE Tracker (Crescente et al., 2018). Long Terminal Repeat (LTR) elements are less common but occupy a larger proportion of the genome, and were identified using the GenomeTools package (Gremme et al., 2013). To reduce the number of false positive LTR transposons, only those that contained PPT (poly purine extract) or PBS (primer binding sites) were kept and the rest filtered. We further filtered the LTR candidates to eliminate three main sources of false positives: tandem local repeats such as centromeric repeats, local gene clusters derived from recent gene duplications, and two other transposable elements located in adjacent regions. We also identified elements with nested insertions. After processing known MITEs and LTR elements, we identified additional repetitive sequences using RepeatModeler against a transposase database and excluded gene fragments using ProtExcluder.

The annotation pipeline maker (Campbell et al. 2014) was used to identify gene models and predict functional annotations in the draft genome. Both est2genome and protein2genome modes were used initially to make $a b$ initio gene predictions from EST and protein evidence, respectively. The EST evidence was based on our own transcriptome data whereas the protein evidence was gathered from the reference proteomes of six closely related plants available from the SwissProt database: Arabidopsis thaliana, Glycine max, Brassica oleracea, Medicago truncatula, Brassica napus, and Brassica rapa. From the first round of annotation, high confidence models were predicted by the maker2zff command with default minimums ( $50 \%$ of splice sites confirmed by EST alignment, $50 \%$ of exons match an EST alignment, $50 \%$ of exons overlap any evidence, and maximum AED of 0.5 ). These predictions were used to train the $a b$ initio gene predictor snap (Korf, 2004). A second round of maker was run using the hidden Markov model (HMM) from snap rather than the est2genome mode. All other settings were the same as for the first run, with the transcripts now being used only as evidence to support $a b$ initio gene predictions. Two more rounds of annotation and gene prediction improvement followed. An Augustus gene prediction file was also generated for use as a second $a b$ initio prediction (Stanke and Morgenstern, 2005). For the final round of annotation in maker, the final HMM file from snap, the $A$. petiolata species Augustus library and gene prediction file were all used in addition to the following settings: always complete, single exons, and using correct EST fusion.

We used Benchmarking Universal Single-Copy Orthologs (BUSCO) v4.1 (Simão et al., 2015) to assess the assembly quality of the final draft genome. We used plant lineage delineation from the EmbryophytaDB V10 database, focusing on universal orthologs present in $>90 \%$ of lineages, resulting in a total of 1,440 BUSCO orthologs. We used minimap2 check for assembly contiguity and synteny with the model plant Arabidopsis thaliana using the TAIR 10 assembly with up to $20 \%$ sequence divergence. Plots generated with the R package pafr.

The genome annotation files were then curated through deFusion to resolve fused gene annotation problems (Wang, 2020), as well as EvidenceModeler (Haas et al., 2008) to combine $a b$ initio gene predictions and protein and transcript alignments into weighted consensus gene structures. The 
functional annotations were then created using NCBI BLAST+ and InterProScan (Jones et al., 2014) by adding new names, domains, and putative functions to improve the utility of the genome database.

\section{Data Availability}

Raw data used for genome assembly, transcriptome assembly, and the final draft genome can be found in the NCBI SRA database (accession numbers TBD) under BioProject SUB9096116 (BioSample Accession: SAMN17958863). Analysis scripts are available on GitHub (https://github.com/turkrhen/snapping_turtle_genome_scripts).

\section{Results and Discussion}

Whole Genome Shotgun (WGS) sequencing of genomic DNA produced 45.8Gb from 229 million HiSeq reads and an additional 7.9Gb from 15.8 million MiSeq reads. This represents an estimated $68 \times$ average coverage of the genome with an estimated genome size of $1.07 \mathrm{~Gb}$. This is similar to published size of 1.35Gb estimated by flow cytometry (Barow and Meister, 2002). Initial assembly with ALLPATHS-LG resulted in 16,743 contigs longer than $1 \mathrm{~Kb}$. Technically, these are scaffolded contigs linked using mate pairs, however we refer to these as contigs to avoid confusion with the scaffolds created by merging heterozygous loci in the redundans program. The final assembled genome was $1.08 \mathrm{~Gb}$ long across 694 scaffolds larger than $1 \mathrm{~Kb}$, and more than $50 \%$ of the genome is contained in the ten longest scaffolds (Table 1).

Cytological studies of $A$. petiolata suggest variation in chromosome number and ploidy (Cavers et al., 1979; Weiss-Schneeweiss and Schneeweiss, 2003), and whether the hexaploids are allo- or autopolyploids. Approximately $36 \%$ of contigs $(6,045$ of 16,743$)$ remain heterozygous after redundans assembly; however, average identity within these contigs was $94.8 \%$, meaning that an average of just $2 \%$ of sites in the genome assembly are heterozygous. Similarly, just 3.2\% of BUSCO genes mapped to more than two scaffolds ( 46 of 1440), consistent with a low level of heterozygosity and minimal duplication of housekeeping genes.

This relatively low level of heterozygosity is consistent with a diploid or highly-inbred autopolyploid. A relatively simple genome combined with geographic variation in ploidy make $A$. petiolata an appealing species to study the role of polyploidy in local adaptation and range expansion, which is an active area of research (e.g. (Pandit et al., 2011; Payseur and Rieseberg, 2016; te Beest et al., 2012)).

Table 1. Assembly statistics for the Alliaria petiolata genome

\begin{tabular}{ll} 
Statistic & Value \\
\hline \# contigs (>= 1000b) & 694 \\
\# contigs (>= 50,000b) & 227 \\
Total length & $1,075,010,735$ \\
Total length (>= 50,000b) & $1,071,536,925$ \\
Largest contig & $485,611,451$ \\
GC $(\%)$ & 37.2 \\
$N_{50}$ & $121,941,980$ \\
$N_{75}$ & $40,840,077$ \\
$L_{50}$ & 2
\end{tabular}


$\mathrm{L}_{75}$

Mean Sequence Length
5

$1,549,006.82$

Most (98.7\%) of the essential single-copy genes from BUSCO mapped to our assembly (Fig. 1), with $71.5 \%$ occurring only once in the assembled genome. Similarly, $98 \%$ of sequence reads from the transcriptome experiment mapped successfully to the assembled genome. Sequencing of the transcriptome libraries yielded a total of $68.1 \mathrm{~Gb}$ from 272.5 million paired reads. Trimming sequence reads for quality reduced usable data by less than $2 \%$.

Complete (C) and single-copy (S)

Complete (C) and duplicated (D)

Missing (M)

Fragmented $(\mathrm{F})$

\section{ALLPATHS-LG}

C:1327 [S:508, D:819], F:27, M:86, n:1440

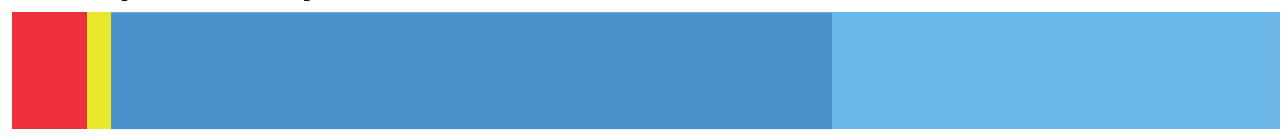

REDUNDANS

C:1370 [S:980, D:390], F:27, M:43, n:1440

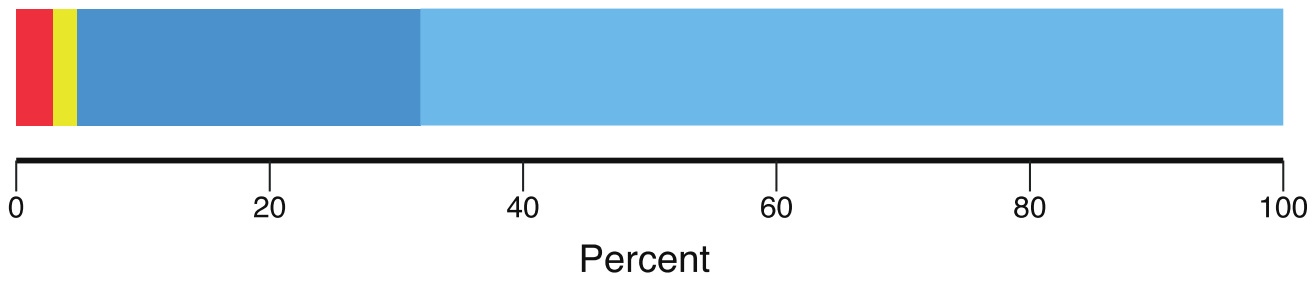

Our de novo transcriptome assembly included 699,048 putative isoform "transcripts" representing $535 \mathrm{Mb}$ with $\mathrm{N} 50$ of 1,233 base pairs. The minimum transcript length was 201 as set by the Trinity default parameter while 1382 ( 1.98\%) of transcripts were longer than $5 \mathrm{~Kb}$. These transcripts clustered into 350,672 hypothetical genes with an average of 2.18 isoforms and 26,910 ( 7.67\%) of hypothetical genes having more than five isoforms. A BLAST search of hypothetical genes to

Figure 1. Percentage of predicted single-copy plant genes from BUSCO that are found one (light blue) or more times (dark blue), or are missing (red) or fragmented (yellow) in the annotated genome assembly of Alliaria petiolata. the SwissProt protein database matched 10,352 proteins with at least $90 \%$ coverage of the query sequence, including 7,930 proteins with $100 \%$ coverage (Table 2 ).

Our TE annotation analysis identified 8,220 unique sequences across the $A$. petiolata genome. Of these, 112 were classified as LTRs with relatively recent origin ( $99 \%$ similarity), 240 as relatively old (85\%), and 7,137 were classified as miniature inverted transposable elements (MITE). An additional 731 sequences were found to match the DNA transposase family. After masking TE sequences the final gene set from

278 (Table 2). 
A dot-plot comparison of gene synteny with the model plant Arabidopsis thaliana (TAIR 10) revealed large blocks of orthologous sequence (Figure 2). However, the arrangement of synteny blocks shows a complete re-arrangement of $A$. thaliana chromosomes when mapped to the $A$. petiolata contigs. Despite a high level of asynteny that is characteristic of the Brassicaceae family, the conservation of large synteny blocks can help to identify candidate genes and genetic loci of interest for understanding plant invasions. Future research could also investigate whether gene rearrangements occur among geographically and historically isolated populations of $A$. petiolata, and whether this genomic architecture has played an important role in the spread of the species.

287

Table 2. Summary statistics of genes annotated for the Alliaria petiolata genome assembly.

\begin{tabular}{ll} 
Statistic & Value \\
\hline Number of genes & 64,770 \\
Number of exons & 408,155 \\
Number of introns & 343,385 \\
Overlapping genes & 9,669 \\
Contained genes & 1,464 \\
Total gene length & $210,804,785$ \\
Total exon length & $102,316,121$ \\
Total intron length & $109,175,434$ \\
Total CDS length & 842,788 \\
\% of genome covered by genes & 19.6 \\
\% of genome covered by CDS & 7.8 \\
Mean mRNAs per gene & 1 \\
Mean exons per mRNA & 6 \\
Mean introns per mRNA & 5 \\
Mean gene length & 3,255 \\
Mean intron length & 318 \\
Mean exon length & 251 \\
Mean CDS length & 1301
\end{tabular}




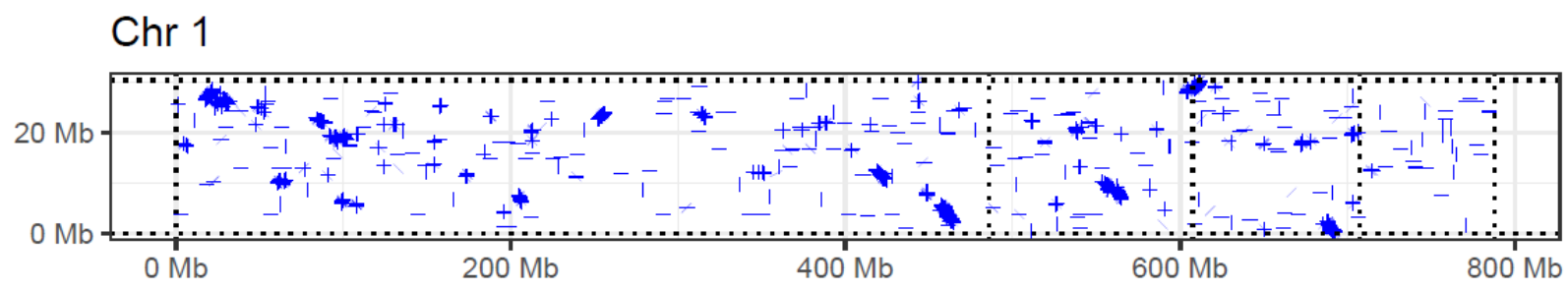

\section{Chr 2}

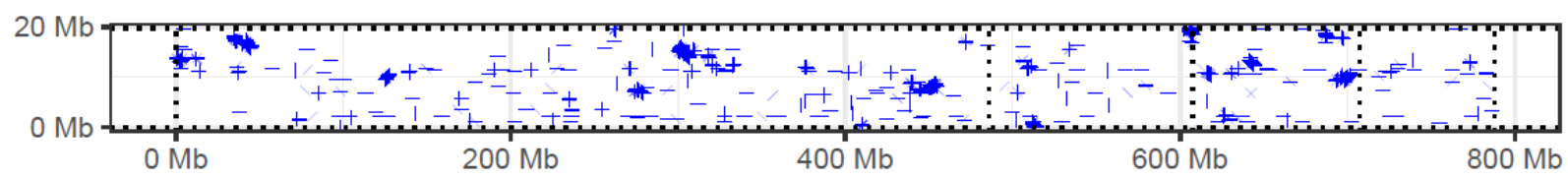

\section{Chr 3}

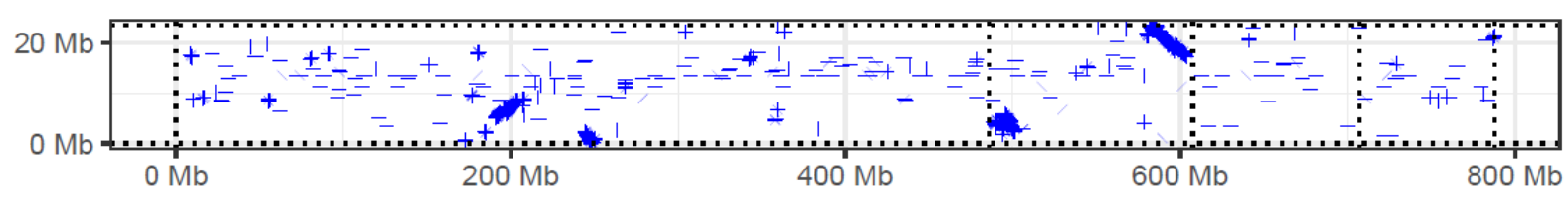

\section{Chr 4}

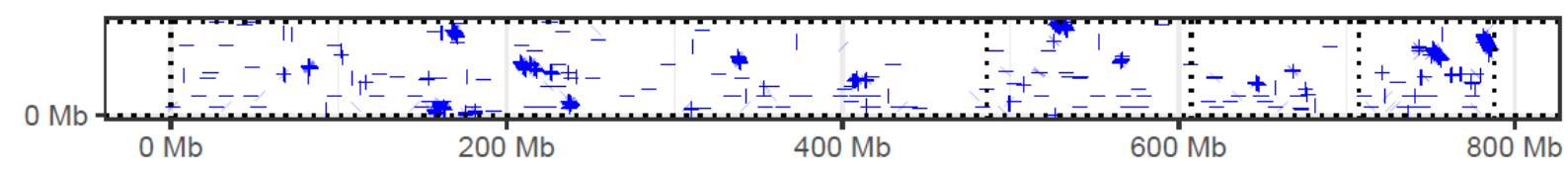

\section{Chr 5}

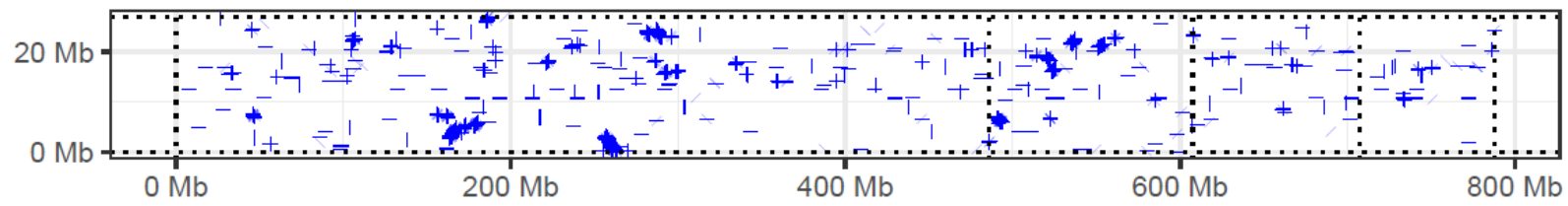

A. petiolata

294 Figure 2. Dot-plot showing blocks of synteny between scaffolds of the Alliaria petiolata assembly (xaxis), and five chromosomes of the model plant Arabidopsis thaliana. Blue lines show aligned sequences with up to $20 \%$ divergence. Vertical dotted lines denote separation of the major scaffolds of the $A$. petiolata assembly.

\section{Conclusions}

299 There is a growing interest in the genetic causes and consequences of range expansion and biological invasion. The field of invasion genetics has emerged from ecological and evolutionary studies of invasive species but lacks well-developed model systems. The draft genome and gene annotation reported here represents an important link from the many field and experimental studies of $A$. petiolata to the genetic architecture of adaptation and invasion. High levels of self-fertility and the resultant low levels heterozygosity observed in the genome will be beneficial for future projects linking ecologically important phenotypes to specific genes. The genomic resources reported here complement available seed resources, experimental findings, and field data to accelerate genomic and molecular studies of $A$. petiolata as a candidate for a model system in invasion genetics. 


\section{Acknowledgements}

The authors are grateful for bioinformatics support from C Grassa, J Stafford, and H Schmider, hardware support from C MacPhee, and wetlab assistance from M Todesco, W Chen and A Siew. We also thank M Todesco and D Galanti for comments that improved the manuscript. This work was supported by NSERC Discovery grants to RIC and LHR.

\section{Literature Cited}

Andrews, S., 2010. FastQC A Quality Control tool for High Throughput Sequence Data [Online] [WWW Document]. URL http://www.bioinformatics.babraham.ac.uk/projects/fastqc/ (accessed 11.19.20).

Anthony, M.A., Frey, S.D., Stinson, K.A., 2017. Fungal community homogenization, shift in dominant trophic guild, and appearance of novel taxa with biotic invasion. Ecosphere 8, e01951. https://doi.org/10.1002/ecs2.1951

Barow, M., Meister, A., 2002. Lack of correlation between AT frequency and genome size in higher plants and the effect of nonrandomness of base sequences on dye binding. Cytometry 47, 1-7. https://doi.org/10.1002/cyto.10030

Barrett, S.C.H., 2015. Foundations of invasion genetics: The Baker and Stebbins legacy. Molecular Ecology 24, 1927-1941. https://doi.org/10.1111/mec.13014

Barrett, S.C.H., Colautti, R.I., Dlugosch, K.M., Rieseberg, L.H., 2016. Invasion Genetics: The Baker and Stebbins Legacy. Wiley.

Bock, D.G., Caseys, C., Cousens, R.D., Hahn, M.A., Heredia, S.M., Huebner, S., Turner, K.G., Whitney, K.D., Rieseberg, L.H., 2015. What we still don't know about invasion genetics. Molecular Ecology 24, 2277-2297. https://doi.org/10.1111/mec.13032

Boheemen, L.A. van, Lombaert, E., Nurkowski, K.A., Gauffre, B., Rieseberg, L.H., Hodgins, K.A., 2017. Multiple introductions, admixture and bridgehead invasion characterize the introduction history of Ambrosia artemisiifolia in Europe and Australia. Molecular Ecology 26, 5421-5434. https://doi.org/10.1111/mec.14293

Bossdorf, O., Prati, D., Auge, H., Schmid, B., 2004. Reduced competitive ability in an invasive plant. Ecology Letters 7, 346-353. https://doi.org/10.1111/j.1461-0248.2004.00583.x

Bourne, S.D., Hudson, J., Holman, L.E., Rius, M., 2020. Marine Invasion Genomics: Revealing Ecological and Evolutionary Consequences of Biological Invasions, in: Oleksiak, M.F., Rajora, O.P. (Eds.), Population Genomics: Marine Organisms, Population Genomics. Springer International Publishing, Cham, pp. 363-398. https://doi.org/10.1007/13836_2018_21

Callaway, R.M., Cipollini, D., Barto, K., Thelen, G.C., Hallett, S.G., Prati, D., Stinson, K., Klironomos, J., 2008. Novel weapons: Invasive plant suppresses fungal mutualists in America but not in its native Europe. Ecology 89, 1043-1055. https://doi.org/10.1890/07-0370.1

Cavers, P.B., Heagy, M.I., Kokron, R.F., 1979. The Biology of Canadian Weeds: 35. Alliaria petiolata (M. Bieb.) Cavara and Grande. Canadian Journal of Plant Science 59, 217-229.

Cipollini, D., Cipollini, K., 2016. A review of garlic mustard (Alliaria petiolata, Brassicaceae) as an allelopathic plant. tbot 143, 339-348. https://doi.org/10.3159/TORREY-D-15-00059

Clarke, J.D., 2009. Cetyltrimethyl Ammonium Bromide (CTAB) DNA Miniprep for Plant DNA Isolation. Cold Spring Harb Protoc 2009, pdb.prot5177. https://doi.org/10.1101/pdb.prot5177

Colautti, R., Franks, S.J., Hufbauer, R.A., Kotanen, P.M., Torchin, M., Byers, J.E., Pyšek, P., Bossdorf, O., 2014. The Global Garlic Mustard Field Survey (GGMFS): challenges and opportunities of a unique, large-scale collaboration for invasion biology. NeoBiota 21, 29-47. https://doi.org/10.3897/neobiota.21.5242 
Crescente, J.M., Zavallo, D., Helguera, M., Vanzetti, L.S., 2018. MITE Tracker: an accurate approach to identify miniature inverted-repeat transposable elements in large genomes. BMC Bioinformatics 19, 348. https://doi.org/10.1186/s12859-018-2376-y

Dlugosch, K.M., Parker, I.M., 2008. Invading populations of an ornamental shrub show rapid life history evolution despite genetic bottlenecks. Ecol. Lett. 11, 701-709. https://doi.org/10.1111/j.14610248.2008.01181.x

Duchesneau, K., Golemiec, A., Colautti, R.I., Antunes, P.M., 2020. Functional shifts of soil microbial communities associated with Alliaria petiolata invasion. bioRxiv 2020.08.13.248849. https://doi.org/10.1101/2020.08.13.248849

Esmailbegi, S., Al-Shehbaz, I.A., Pouch, M., Mandáková, T., Mummenhoff, K., Rahiminejad, M.R., Mirtadzadini, M., Lysak, M.A., 2018. Phylogeny and systematics of the tribe Thlaspideae (Brassicaceae) and the recognition of two new genera. TAXON 67, 324-340. https://doi.org/10.12705/672.4

Gnerre, S., MacCallum, I., Przybylski, D., Ribeiro, F.J., Burton, J.N., Walker, B.J., Sharpe, T., Hall, G., Shea, T.P., Sykes, S., Berlin, A.M., Aird, D., Costello, M., Daza, R., Williams, L., Nicol, R., Gnirke, A., Nusbaum, C., Lander, E.S., Jaffe, D.B., 2011. High-quality draft assemblies of mammalian genomes from massively parallel sequence data. PNAS 108, 1513-1518. https://doi.org/10.1073/pnas.1017351108

Grabherr, M.G., Haas, B.J., Yassour, M., Levin, J.Z., Thompson, D.A., Amit, I., Adiconis, X., Fan, L., Raychowdhury, R., Zeng, Q., Chen, Z., Mauceli, E., Hacohen, N., Gnirke, A., Rhind, N., di Palma, F., Birren, B.W., Nusbaum, C., Lindblad-Toh, K., Friedman, N., Regev, A., 2011. Trinity: reconstructing a full-length transcriptome without a genome from RNA-Seq data. Nat Biotechnol 29, 644-652. https://doi.org/10.1038/nbt.1883

Gremme, G., Steinbiss, S., Kurtz, S., 2013. GenomeTools: A Comprehensive Software Library for Efficient Processing of Structured Genome Annotations. IEEE/ACM Transactions on Computational Biology and Bioinformatics 10, 645-656. https://doi.org/10.1109/TCBB.2013.68

Haas, B.J., Papanicolaou, A., Yassour, M., Grabherr, M., Blood, P.D., Bowden, J., Couger, M.B., Eccles, D., Li, B., Lieber, M., MacManes, M.D., Ott, M., Orvis, J., Pochet, N., Strozzi, F., Weeks, N., Westerman, R., William, T., Dewey, C.N., Henschel, R., LeDuc, R.D., Friedman, N., Regev, A., 2013. De novo transcript sequence reconstruction from RNA-seq using the Trinity platform for reference generation and analysis. Nature Protocols 8, 1494-1512. https://doi.org/10.1038/nprot.2013.084

Haas, B.J., Salzberg, S.L., Zhu, W., Pertea, M., Allen, J.E., Orvis, J., White, O., Buell, C.R., Wortman, J.R., 2008. Automated eukaryotic gene structure annotation using EVidenceModeler and the Program to Assemble Spliced Alignments. Genome Biology 9, R7. https://doi.org/10.1186/gb2008-9-1-r7

Haribal, M., Yang, Z., Attygalle, A.B., Renwick, J.A.A., Meinwald, J., 2001. A Cyanoallyl Glucoside from Alliaria petiolata, as a Feeding Deterrent for Larvae of Pieris napi oleracea. J. Nat. Prod. 64, 440443. https://doi.org/10.1021/np000534d

Jones, P., Binns, D., Chang, H.-Y., Fraser, M., Li, W., McAnulla, C., McWilliam, H., Maslen, J., Mitchell, A., Nuka, G., Pesseat, S., Quinn, A.F., Sangrador-Vegas, A., Scheremetjew, M., Yong, S.-Y., Lopez, R., Hunter, S., 2014. InterProScan 5: genome-scale protein function classification. Bioinformatics 30, 1236-1240. https://doi.org/10.1093/bioinformatics/btu031

Korf, I., 2004. Gene finding in novel genomes. BMC Bioinformatics 5, 59. https://doi.org/10.1186/14712105-5-59

Langmead, B., Salzberg, S.L., 2012. Fast gapped-read alignment with Bowtie 2. Nature Methods 9, 357359. https://doi.org/10.1038/nmeth.1923 
Lankau, R.A., Nuzzo, V., Spyreas, G., Davis, A.S., 2009. Evolutionary limits ameliorate the negative impact of an invasive plant. Proc. Natl. Acad. Sci. U. S. A. 106, 15362-15367. https://doi.org/10.1073/pnas.0905446106

Lewis, K.C., Bazzaz, F.A., Liao, Q., Orians, C.M., 2006. Geographic patterns of herbivory and resource allocation to defense, growth, and reproduction in an invasive biennial, Alliaria petiolata. Oecologia 148, 384-395. https://doi.org/10.1007/s00442-006-0380-9

Mack, R.N., Simberloff, D., Lonsdale, W.M., Evans, H., Clout, M., Bazzaz, F.A., 2000. Biotic invasions: Causes, epidemiology, global consequences, and control. Ecological Applications 10, 689-710.

Martin, M., 2011. Cutadapt removes adapter sequences from high-throughput sequencing reads. EMBnet.journal 17, 10-12. https://doi.org/10.14806/ej.17.1.200

Mooney, H.A., Cleland, E.E., 2001. The evolutionary impact of invasive species. PNAS 98, 5446-5451. https://doi.org/10.1073/pnas.091093398

Nuzzo, V., 1993. Distribution and spread of the invasive biennial Alliaria petiolata (garlic mustard) in North America., in: McKnight, B. (Ed.), Biological Pollution: The Control and Impact of Invasive Exotic Species. Indiana Academy of Science, Indianapolis, Indiana, pp. 137-145.

Pandit, M.K., Pocock, M.J.O., Kunin, W.E., 2011. Ploidy influences rarity and invasiveness in plants. Journal of Ecology 99, 1108-1115. https://doi.org/10.1111/j.1365-2745.2011.01838.x

Payseur, B.A., Rieseberg, L.H., 2016. A genomic perspective on hybridization and speciation. Molecular Ecology 25, 2337-2360. https://doi.org/10.1111/mec.13557

Prati, D., Bossdorf, O., 2004. Allelopathic inhibition of germination by Alliaria petiolata (Brassicaceae). American Journal of Botany 91, 285-288. https://doi.org/10.3732/ajb.91.2.285

Pryszcz, L.P., Gabaldón, T., 2016. Redundans: an assembly pipeline for highly heterozygous genomes. Nucleic Acids Res 44, e113-e113. https://doi.org/10.1093/nar/gkw294

Sax, D.F., Stachowicz, J.J., Gaines, S.D., 2005. Species Invasions: Insights into Ecology, Evolution, and Biogeography. Sinauer.

Simão, F.A., Waterhouse, R.M., loannidis, P., Kriventseva, E.V., Zdobnov, E.M., 2015. BUSCO: assessing genome assembly and annotation completeness with single-copy orthologs. Bioinformatics 31 , 3210-3212. https://doi.org/10.1093/bioinformatics/btv351

Stanke, M., Morgenstern, B., 2005. AUGUSTUS: a web server for gene prediction in eukaryotes that allows user-defined constraints. Nucleic Acids Res 33, W465-W467. https://doi.org/10.1093/nar/gki458

Stinson, K., Kaufman, S., Durbin, L., Lowenstein, F., 2007. Impacts of garlic mustard Invasion on a forest understory community. nena $14,73-88$. https://doi.org/10.1656/10926194(2007)14[73:IOGMIO]2.0.CO;2

te Beest, M., Le Roux, J.J., Richardson, D.M., Brysting, A.K., Suda, J., Kubešová, M., Pyšek, P., 2012. The more the better? The role of polyploidy in facilitating plant invasions. Ann Bot 109, 19-45. https://doi.org/10.1093/aob/mcr277

USDA, N., 2020. The PLANTS Database (http://plants.usda.gov, 10 April 2020). National Plant Data Team, Greensboro, NC.

Wang, J., 2020. wjidea/defusion.

Weiss-Schneeweiss, H., Schneeweiss, G.M., 2003. Karyological investigations of selected angiosperms from Georgia and Azerbaijan. Acta Biologica Cracoviensia Series Botanica 45, 49-56. 